\title{
Type IV collagen turnover is predictive of mortality in COPD: a comparison to fibrinogen in a prospective analysis of the ECLIPSE cohort
}

Sarah Rank Rønnow ${ }^{1,2^{*}+}$, Jannie Marie Bülow Sand ${ }^{1+}$, Lasse Løcke Langholm¹,3, Tina Manon-Jensen ${ }^{1}$, Morten Asser Karsdal ${ }^{1}$, Ruth Tal-Singer ${ }^{4}$, Bruce E. Miller ${ }^{4}$, Jørgen Vestbo ${ }^{5}$ and Diana Julie Leeming ${ }^{1}$

\begin{abstract}
Background: Identifying subjects with chronic obstructive pulmonary disease (COPD) at high risk of exacerbation and mortality is key to aid individual management of COPD. The only FDA approved bloodbased drug development biomarker for patients at high risk of mortality, is plasma fibrinogen. In this study, we benchmarked two biomarkers of basement membrane remodeling, a characteristic of COPD, against plasma fibrinogen alone and as a combination. The biomarkers of basement membrane remodeling are two neoepitopes from of the alpha 3 chain of type IV collagen (COL4A3).

Materials and methods: COL4A3 degradation was assessed by the biomarkers C4Ma3 and tumstatin (TUM) in year 1 plasma samples in 984 COPD subjects, 95 non-smoking controls and 95 smoking controls from the Evaluation of COPD Longitudinally to Identify Predictive Surrogate End-points (ECLIPSE) cohort. They were measured by competitive ELISA using monoclonal antibodies recognizing two specific MMP-generated cleavage site within COL4A3. The level of fibrinogen was previously assessed in year 1 plasma.

Results: In COPD subjects, plasma C4Ma3 levels were significantly correlated with plasma fibrinogen levels (0.389 $(P<0.0001))$. Cox proportional-hazards regression adjusted for relevant confounders showed that high levels of plasma C4Ma3, but not TUM, were related to a higher risk of mortality (hazard ratio $5.12(95 \% \mathrm{Cl} 2.28-11.50)$, $P<0.0001$ ). High levels of plasma fibrinogen were not associated with all-cause mortality in this subpopulation, contradictory to published results. Whereas plasma C4Ma3 multiplied by fibrinogen showed to be related to a higher risk of mortality (hazard ratio $5.74(95 \% \mathrm{Cl} 2.65-12.41), P<0.0001)$. Plasma C4Ma3 levels were related to the number of hospitalizations due to COPD exacerbations in the year before study start $(P=0.0375)$. Fibrinogen levels were related to hospitalized exacerbations prior to study start $(P=0.0058)$ and were also related to future exacerbations $(P<0.0001)$.

\footnotetext{
* Correspondence: Sar@nordicbio.com

†Sarah Rank Rønnow and Jannie Marie Bülow Sand contributed equally to this work.

${ }^{1}$ Nordic Bioscience A/S, Herlev, Denmark

${ }^{2}$ University of Southern Denmark, The Faculty of Health Science, Odense,

Denmark

Full list of author information is available at the end of the article
}

(c) The Author(s). 2019 Open Access This article is distributed under the terms of the Creative Commons Attribution 4.0 International License (http://creativecommons.org/licenses/by/4.0/), which permits unrestricted use, distribution, and reproduction in any medium, provided you give appropriate credit to the original author(s) and the source, provide a link to the Creative Commons license, and indicate if changes were made. The Creative Commons Public Domain Dedication waiver (http://creativecommons.org/publicdomain/zero/1.0/) applies to the data made available in this article, unless otherwise stated. 
(Continued from previous page)

Conclusion: We compared herein fibrinogen, C4Ma3 and TUM as biomarkers for COPD prognosis. Fibrinogen was related to future exacerbation, whereas C4Ma3 and the combination of C4Ma3 with fibrinogen were superior to fibrinogen alone in predicting mortality. This pilot study suggests that the assessment of plasma C4Ma3 could be important for identifying COPD patients with a poor prognosis.

Trial registration: NCT00292552, GSK Study No. SCO104960.

Keywords: COPD, Biomarkers, Basement membrane, Collagen type IV, ECLIPSE

\section{Background}

Chronic obstructive pulmonary disease (COPD) is the third leading cause of death worldwide [1]. It is characterized by progressive airflow limitation related to chronic inflammation, lung tissue destruction and small airway fibrosis. Identifying patients at risk of progression as defined by a rapid decline in forced expiratory volume in one second $\left(\mathrm{FEV}_{1}\right)$, an increased rate of exacerbations or mortality is challenging but crucial for precision medicine. Plasma fibrinogen was the first blood-based biomarker of COPD to be qualified approved by the U.S. Food and Drug Administration (FDA) in 2015 for the enrichment of subjects with COPD at higher risk of progression in clinical trials [2,3]. The approval was based on data from the ECLIPSE cohort, showing its value as a prognostic biomarker to identify COPD subjects at risk of exacerbations or all-cause mortality.

One of the main structural characteristics of COPD is changes to the lung tissue due to altered extracellular matrix (ECM) content and remodeling of the ECM. In the distal airways and alveoli, the ECM is mainly represented by the basement membrane (BM) with only a thin layer of or no interstitial matrix. The ECM layer is thin, thus ensuring easy transport of gases between the air and the blood [4-6], and the alveoli contain a specialized BM that is optimal for this gas transport. There are six distinct type IV collagen $\alpha$-chains, namely $\alpha 1$ (IV) through $\alpha 6$ (IV), which are selectively expressed in different membranes at various stages of development and in specific tissues [5, 7]. The $\alpha 1 \alpha 1 \alpha 2$ (IV) isoform is ubiquitous, but the alveolar BM, as well as the glomerular BM, also contains the specialized $\alpha 3 \alpha 4 \alpha 5$ (IV) isoform that facilitates gas exchange [8, 9]. The function of type IV collagen in both lungs and kidneys indicates that this specialized isoform is important for the diffusion across the BM barrier in these two organs. The $\alpha 3 \alpha 4 \alpha 5$ (IV) isoform has been shown to contain a high number of disulfide cross-links and consequently to be more resistant to proteolytic degradation than $\alpha 1 \alpha 1 \alpha 2$ (IV). Thus, the $\alpha 3 \alpha 4 \alpha 5$ (IV) may be better suited for the more vulnerable position in the alveolar BM [10]. This makes $\alpha 3 \alpha 4 \alpha 5$ (IV) a very specific collagen type IV isoform of the lungs.

Alterations in the BM influence the processes of lung injury and repair, implying an important role for type IV collagen in pulmonary diseases. For example, the disruption of the alveolar BM as a result of matrix metalloproteinase (MMP) activity is a consistent finding in idiopathic pulmonary fibrosis (IPF) [11], that may contribute to aberrant lung remodeling by hindering re-epithelialization and enhancing fibroblast invasion mediated by increasing permeability [12]. Thus, the remodeling of type IV collagen may also be crucial in the pathogenesis of COPD.

Fibrinogen is a coagulation factor that is essential for the blood clotting process in tissues following injury. Plasma levels of fibrinogen have been shown to predict acute exacerbations of COPD as well as all-cause mortality [2, 3]. However, plasma fibrinogen levels may rise in response to extrapulmonary injury and systemic inflammation in COPD which makes it less specific for disease activity specifically in the lung.

In this study, we investigated BM remodeling as a risk biomarker for COPD disease progression as defined by alterations in lung tissue structure. More specifically, we evaluated the alveolar BM remodeling by measuring the serological biomarker (C4Ma3) reflecting MMP-mediated degradation of the type IV collagen alpha 3 chain and tumstatin (TUM) the C-terminal non-collagenous (NC1) domain of the type IV collagen alpha 3 chain. BM remodeling may be more specific for the changes that occur in the lung of COPD patients as compared to plasma fibrinogen and therefore potentially a better prognostic biomarker alone or incremental to the prognostic value of fibrinogen. To investigate this, we benchmarked the performance of BM remodeling against plasma fibrinogen and a combination of plasma C4Ma3 and fibrinogen in relation to acute exacerbations and mortality outcome in subjects with COPD. We conducted a retroprospective analysis of the plasma C4Ma3, TUM and fibrinogen in a subset of the three-year Evaluation of COPD Longitudinally to Identify Predictive Surrogate End-points (ECLIPSE) study.

\section{Methods}

\section{Study design}

The study design of ECLIPSE (clinicaltrials.gov identifier NCT00292552; GSK study code SCO104960) has been described in detail previously [13, 14]. Briefly, ECLIPSE 
is an observational, longitudinal study which evaluated participants at baseline and at months three, six, and subsequently every six months for three years. For the current analyses, we used clinical and biomarker data obtained at year one and year three. Death was recorded up to day 1060 in year three of the study. Cause of death was not available for the present analyses. ECLIPSE complies with the Declaration of Helsinki and Good Clinical Practice Guidelines and has been approved by the ethics committees of the participating centers. All participants provided written informed consent before the performance of any study-related assessments.

\section{Population}

The full ECLIPSE study included 2164 participants diagnosed with COPD based on a post-bronchodilator $\mathrm{FEV}_{1}<80 \%$ of the reference value, $\mathrm{FEV}_{1} /$ forced vital capacity $(\mathrm{FVC}) \leq 0.7$, and a smoking history of $\geq 10$ pack-years [13, 14]. The cohort was recruited from the outpatient clinics of the participating centers. The current study was performed on a subpopulation of 1000 COPD subjects of the ECLIPSE cohort composed of 500 subjects progressing the most and 500 subjects progressing the least as defined by $\mathrm{FEV}_{1}$ decline over the three-year study period. Individuals with available plasma $\mathrm{C} 4 \mathrm{Ma} 3$ data were included in the analyses, giving a total of 984 COPD participants (Fig. 1a). Additionally, plasma C4Ma3 data for smoking $(n=95)$ and never-smoking $(n=95)$ controls age gender and body mass index (BMI) matched to 95 of the COPD subjects were included in the analyses (Fig. 1).

\section{Measurements of serological biomarkers}

Plasma (heparin anticoagulant) samples were collected from participants in the fasting state at year 1 by venipuncture into vacutainer tubes followed by centrifugation at $2000 \mathrm{~g}$ for $10-15 \mathrm{~min}$. Plasma was stored at $-80^{\circ} \mathrm{C}$ until analyzed. Plasma C4Ma3 and TUM levels were measured by validated competitive ELISAs utilizing monoclonal antibodies targeting a specific neo-epitope of type IV collagen generated by MMP cleavage of the alpha 3 chain (Nordic Bioscience, Herlev, Denmark) [15, 16]. Measurements were performed in a blinded manner according to the manufacturer's instructions. Analytes detected by this assay are stable in plasma samples that have undergone at least four freeze/thaw cycles [15]. Prior to this study, plasma fibrinogen levels were measured in year one plasma (dry ethylenediaminetetraacetic acid anticoagulant) samples as described previously [17]. As measurements were performed in plasma and not in serum, in order to be able to compare fibrinogen results to the typically used liquid sodium citrate anti-coagulant, the published cut-off level, plasma fibrinogen levels were corrected by $-13.6 \%$, as described in the statistical analysis plan from the COPD Biomarkers Qualification Consortium for their FDA application (Supplements for [18]).

\section{Statistical analysis}

Basic demographics were compared using Mann-Whitney U test, Kruskal-Wallis test, or chi-squared test as appropriate. Plasma C4Ma3, TUM and fibrinogen levels were compared between groups or with clinical parameters using Spearman's rank correlation, Mann-Whitney U test or Kruskal-Wallis test as appropriate.
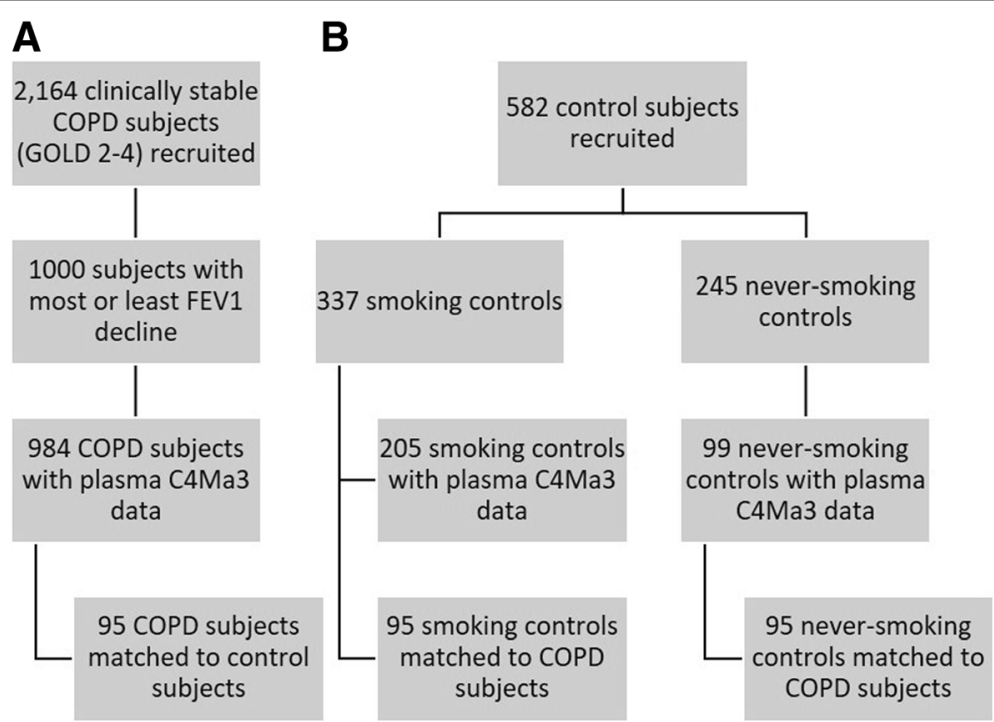

Fig. 1 Study recruitment and participant flow. a COPD participants. b controls 
The association of plasma C4Ma3 and plasma fibrinogen with future and prior acute exacerbations was investigated by logistic regression.

Cox proportional hazards analyses were used to assess the prognostic value of plasma C4Ma3, TUM and fibrinogen levels for all-cause mortality for one standard deviation (SD) increase in the biomarker. Plasma C4Ma3 data were dichotomized based on receiver operating curve (ROC) analyses. The ROC curve had an area under the curve (AUC) of 0.68 (95\% CI 0.65-0.71), $P=0.0005$, and gave a cut-off of $5.5 \mathrm{ng} / \mathrm{mL}$ for the identification of subjects who died with a sensitivity of 40.0 and specificity of 90.8. Plasma fibrinogen data were dichotomized based on the published cut-off level of $350 \mathrm{mg} / \mathrm{dL}$. A cut-off value for plasma C4Ma3 multiplied by plasma fibrinogen (C4Ma3*Fibrinogen) was defined by a ROC analysis for identification of COPD subjects at risk of mortality. The ROC curve had an AUC of 0.68 (95\% CI $0.58-0.80, P=0.0004$ ) and gave a cut-off of 2037 for the identification of subjects who died with a sensitivity of 53.3 and specificity of 83.9. Mortality risk for groups below and above the cut-off was compared using Cox proportional hazards analysis with or without relevant confounders as determined by univariate Cox proportional hazards analysis. Kaplan-Meier survival curves compared the mortality risk for patients belonging to the high vs. low biomarker groups. All tests performed (MedCalc Statistical Software version 16.8.4, MedCalc Software bvba, Ostend, Belgium) were two-sided at the 0.05 level of significance, and all $P$ values are nominal as no adjustments were made for multiple comparisons.

\section{Results}

\section{C4Ma3 is increased in COPD patients}

Levels of plasma C4Ma3 were significantly different between COPD and matched control participants
$(P=0.0047)$. Group analysis showed that plasma C4Ma3 levels were significantly elevated in COPD subjects (median 38 pack years) as compared to never-smoking, but not smoking controls (median 30 pack-years) (Table 1/ Fig. 2). Plasma fibrinogen level was significantly increased in COPD subjects compared to smoking controls and never-smoking controls (Table 1/Fig. 2). Whereas, TUM did not show any difference between the three groups (Fig. 2). In COPD subjects, plasma C4Ma3 levels were significantly correlated with plasma fibrinogen levels (Spearman' correlation coefficient $0.389(P<0.0001)$.

\section{Basic demographics}

Basic demographics for the full COPD population are shown in Table 2. Mean age was 63.1 (7.2) years, BMI was 26.8 (5.9), and 357 (36\%) were female. Mean pack-years were 47.4 (26.0) and 363 (37\%) were current smokers. Post-bronchodilator $\mathrm{FEV}_{1}$ was a mean of $50.6 \%$ (15.2) of predicted value, 558 (57\%) had significant emphysema, $439(45 \%)$ reported one or more exacerbation in the year prior to study start, and 30 (3\%) died from year one to the end of the study period (year three).

\section{C4Ma3 may be superior to fibrinogen in predicting mortality}

COPD subjects which were registered as dead as compared to alive at the end of the study period had significantly higher levels of plasma C4Ma3, TUM and fibrinogen $(P=0.0008, P=0.0467$ and $P=0.0006$, respectively) (Table $2 /$ Fig. 3). Univariate Cox proportional hazard regression showed age, smoking status, 6-min walk distance (6MWD), modified Medical Research Council (mMRC) dyspnea score, and prior hospitalizations due to acute exacerbations to influence mortality risk (Table 3). Cox regression analysis adjusted for age, smoking status, prior hospitalizations due to exacerbations, mMRC and 6MWD showed that plasma C4Ma3

Table 1 Demographic characteristics of COPD subjects and controls

\begin{tabular}{|c|c|c|c|c|}
\hline Parameter & Smoking controls & Never-smoking controls & COPD & $P$ value \\
\hline$n$ & 95 & 95 & 95 & \\
\hline Age, mean (SD) & $59.4(7.2)$ & $58.8(7.6)$ & $60.0(6.4)$ & $0.663 ¥$ \\
\hline Female gender, $\mathrm{n}(\%)$ & $47(49 \%)$ & $47(49 \%)$ & $47(49 \%)$ & $1.000 \pm$ \\
\hline BMI, mean (SD) & $27.4(4.3)$ & $28.4(4.5)$ & $27.4(5.5)$ & $0.239 ¥$ \\
\hline Pack-years, median (IQR) & $30(18-40)$ & $0(0-0)$ & $38(30-48)$ & $<0.0001 ¥$ \\
\hline Current smokers, n (\%) & $47(49 \%)$ & $0(0 \%)$ & $47(49 \%)$ & $<0.0001 £$ \\
\hline Cardiovascular history, n (\%) & $26(27 \%)$ & $16(17 \%)$ & $21(22 \%)$ & $0.217 £$ \\
\hline Post bronchodilator FEV 1 (\% predicted), mean (SD) & $110.3(13.6)$ & $114.0(13.7)$ & $53.3(15.6)$ & $<0.0001 ¥$ \\
\hline C4Ma3 (ng/mL), median (IQR) & $3.04(2.44-3.85)$ & $2.76(2.20-3.35)$ & $3.12(2.46-4.22)$ & $0.0047 ¥$ \\
\hline TUM (ng/mL), median (IQR) & $0.71(0.64-0.78)$ & $0.63(0.60-0.73)$ & $0.73(0.63-0.80)$ & $0.263 ¥$ \\
\hline Fibrinogen (mg/dL), median (IQR) & $343.4(311.0-383.6)$ & $326.6(287.7-375.8)$ & $356.4(318.8-421.2)$ & $0.0009 ¥$ \\
\hline
\end{tabular}

$¥$ Kruskal-Wallis; $£$ Chi-squared test 

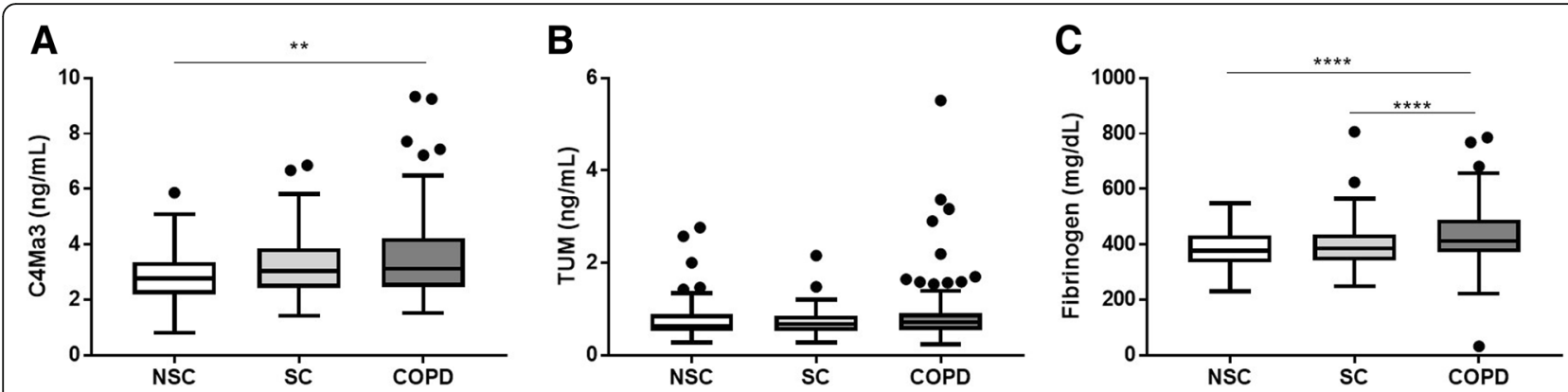

Fig. 2 Plasma C4Ma3 (a), TUM (b) and fibrinogen (c) levels in matched never-smoking controls (NSC), smoking controls (SC), and COPD subjects. Each group $n=95$. Data are shown as Tukey box-and-whiskers plot with a line indicating median, the box indicating the 25th and 75th percentiles (the interquartile range, IQR), and whiskers indicating 1.5 times the IQR. Data points outside this limit are shown by individual data points. Data were analyzed by Kruskal-Wallis test using Dunn's multiple comparison. Asterisks indicate statistical significance: ${ }^{* *} p<0.01$, and ${ }^{* * * *} p<0.0001$

was an independent predictor of mortality with a hazard ratio of 1.57 (95\% CI 1.29-1.92) per SD increase $(P<0.0001)$, whereas TUM showed not to be an independent predictor of mortality. Plasma fibrinogen showed similar results as $\mathrm{C} 4 \mathrm{Ma} 3$ with a hazard ratio of 1.62 (95\% CI $1.31-1.99)$ per SD increase $(P<0.0001)$ (Table 3). Plasma C4Ma3 values were dichotomized into a high and a low group based on a ROC curve, which was analyzed by Kaplan-Meier survival curve (Fig. 4a). For comparison, plasma fibrinogen was analyzed using the published cut-off value of $350 \mathrm{mg} / \mathrm{dL}$ (Fig. 4b) [18]. Cox proportional hazards regression was used for assessing the risk of mortality in high vs low group in a crude and adjusted analysis. The hazard ratio for the high plasma C4Ma3 group was 4.68 (95\% CI $1.40-15.71, P<0.0001)$. Here, $10.3 \%$ of subjects in the high group died while only $2.3 \%$ of the subjects died in the low C4Ma3 group. The hazard ratio for the high plasma fibrinogen group was 3.30 (95\% CI 1.55-7.03, P $=0.0186)$. In the adjusted analysis high C4Ma3 level, age, and former smoking were included in the model while prior hospitalized acute exacerbations, mMRC dyspnea score and 6MWD were not significant. This model provided a hazard ratio for being in the high plasma C4Ma3 group of 5.12 (95\% CI 2.28-11.50, $P=$ $0.0001)$. A similar model with high plasma fibrinogen level showed fibrinogen to be non-significant and instead, the model included age, former smoking and mMRC dyspnea score. In addition, adding both high C4Ma3 and high Fibrinogen to a model only included age, smoking and plasma $\mathrm{C} 4 \mathrm{Ma} 3$ providing a hazard ratio for being high in plasma C4Ma3 of 3.75 (95\% CI 1.60-8.80, $P=0.0024)$. High levels of fibrinogen showed to be non-significant (Table 4).

\section{Combining C4Ma3 and fibrinogen improves prediction of mortality}

To investigate if the added effects of plasma C4Ma3 and fibrinogen could provide additional predictive value, we forced the two biomarkers into a model in addition to age and smoking status. Cox proportional hazards regression was used to assess the risk of mortality in high vs low groups in an adjusted analysis defined by a ROC curve. This model provided a hazard ratio for being in the high plasma $\mathrm{C}_{\mathrm{MMa}}{ }^{*}$ Fibrinogen group of 5.74 (95\% CI 2.65-12.41), $P<0.0001$, which was higher than both plasma C4Ma3 and plasma fibrinogen individually. Age and former smoking were included in the model while prior hospitalized acute exacerbations, mMRC dyspnea score and 6MWD were not. (Table 5).

\section{C4Ma3 is related to hospitalized exacerbations}

Plasma C4Ma3 levels were related to the number of hospitalizations due to COPD exacerbations in the year prior to study start (Kruskal-Wallis $P=0.0375$ ) (Fig. 5a). Levels were not able to predict any future self-reported exacerbations (data not shown). Plasma fibrinogen levels were related to hospitalized exacerbations prior to study start (Kruskal-Wallis $P=0.0058$ ) (Fig. 5b) as well as being related to future exacerbations in the year following biomarker measurement (Kruskal-Wallis $P \geq 0.0001$ ) (data not shown). To find out if high plasma C4Ma3 and high plasma fibrinogen alone were able to predict exacerbations in the following year, a logistic regression was performed. BMI, airflow Obstruction, Dyspnea and Exercise capacity index (BODE) and mMRC dyspnea score were included in the model while age, former smoking, and 6MWD were not significant. Only the BODE index was able to predict exacerbations in the following year. This was also the case when including high $\mathrm{C} 4 \mathrm{Ma} 3 * \mathrm{Fi}$ brinogen in the model.

\section{Discussion}

Identifying patients at highest risk of progression or the highest disease activity and determining responders versus non-responders to a disease-modifying drug in expensive clinical trials would be valuable to COPD 
Table 2 Basic Demographics

\begin{tabular}{|c|c|c|c|c|}
\hline Parameter & COPD & Survivors & Deceased & $P$ value (survivors vs. deceased) \\
\hline $\mathrm{n}$ & 984 & 954 & 30 & \\
\hline Age, mean (SD) & $63.1(7.2)$ & $62.9(7.2)$ & $68.2(6.3)$ & $<0.0001 ¥$ \\
\hline Female gender, n (\%) & $357(36 \%)$ & $346(36 \%)$ & $11(37 \%)$ & $0.964 £$ \\
\hline BMI, mean (SD) & $26.8(5.9)$ & $26.8(5.8)$ & $27.3(7.8)$ & $0.777 ¥$ \\
\hline Pack-years, mean (SD) & $47.4(26.0)$ & $47.1(25.3)$ & $55.9(42.5)$ & $0.934 ¥$ \\
\hline Current smokers, n (\%) & $363(37 \%)$ & $360(38 \%)$ & $3(10 \%)$ & $0.002 £$ \\
\hline Cardiovascular history, n (\%) & $310(32 \%)$ & $298(31 \%)$ & $12(40 \%)$ & $0.309 £$ \\
\hline Post bronchodilator FEV 1 (\% predicted), mean (SD) & $50.6(15.2)$ & $50.6(15.3)$ & $48.5(13.0)$ & $0.384 ¥$ \\
\hline GOLD stage, n (\%) & & & & $0.695 £$ \\
\hline 2 & $487(49 \%)$ & $473(50 \%)$ & $14(47 \%)$ & \\
\hline 3 & $397(40 \%)$ & $383(40 \%)$ & $14(47 \%)$ & \\
\hline 4 & $100(10 \%)$ & $98(10 \%)$ & $2(7 \%)$ & \\
\hline GOLD stage, n (\%) & & & & $0.231 £$ \\
\hline A & $250(25 \%)$ & $244(26 \%)$ & $6(20 \%)$ & \\
\hline B & $128(13 \%)$ & $121(13 \%)$ & $7(23 \%)$ & \\
\hline C & $226(23 \%)$ & $222(23 \%)$ & $4(13 \%)$ & \\
\hline D & $339(34 \%)$ & $329(34 \%)$ & $10(33 \%)$ & \\
\hline mMRC score, median (IQR) & $1(1-2)$ & $1(1-2)$ & $2(1-3)$ & $0.022 ¥$ \\
\hline SGRQ-C total score, median (IQR) & $47.2(33.2-60.6)$ & $47.2(33.2-60.2)$ & $53.3(33.0-66.0)$ & $0.294 ¥$ \\
\hline 6MWD (meters), mean (SD) & $385.4(119.0)$ & $387.0(119.1)$ & $335.3(105.0)$ & $0.013 ¥$ \\
\hline FV950 baseline, mean (SD) & $16.3(11.3)$ & $16.2(11.3)$ & $17.4(10.5)$ & $0.425 ¥$ \\
\hline Emphysema (\%LAA $\geq 10 \%), n(\%)$ & $558(57 \%)$ & $538(56 \%)$ & $20(67 \%)$ & $0.264 £$ \\
\hline $\begin{array}{l}\text { Number of hospitalized exacerbations in year } \\
\text { prior to study, mean (SD) }\end{array}$ & $0.16(0.53)$ & $0.16(0.52)$ & $0.37(0.85)$ & $0.036 ¥$ \\
\hline BODE index, median (IQR) & $3.0(1.0-4.0)$ & $3.0(1.0-4.0)$ & $3.5(2-5)$ & $0.065 ¥$ \\
\hline Dead by end of study, n (\%) & $30(3 \%)$ & $0(0 \%)$ & $30(100 \%)$ & $<0.0001 £$ \\
\hline Survival time (days), mean (SD) & $1056(28)$ & $1060(0)$ & $922(84)$ & $<0.0001 ¥$ \\
\hline C4Ma3 (ng/mL), median (IQR) & $3.28(2.55-4.33)$ & $3.25(2.54-4.30)$ & $4.02(3.16-6.05)$ & $0.0008 ¥$ \\
\hline TUM (ng/mL), median (IQR) & $0.71(0.69-0.74)$ & $0.71(0.69-0.73)$ & $0.79(0.71-1.17)$ & $0.0467 ¥$ \\
\hline Fibrinogen (mg/dL), median (IQR) & $378.4(334.4-434.1)$ & $377.6(333.5-432.0)$ & $445.4(359.4-527.0)$ & $0.0006 ¥$ \\
\hline
\end{tabular}

$¥$ Mann-Whitney; $£$ Chi-squared test. Data are shown as mean $\pm S D$, median (25th, 75th) or number (\%). BMI body mass index, FEV ${ }_{1}$ post-bronchodilator forced expiratory volume in $1 \mathrm{~s}$, GOLD global initiative for chronic obstructive lung disease, $m M R C$ modified Medical Research Council dyspnea score, SGRQ St. George's respiratory questionnaire, $6 M W D$ 6-min walk distance, \%LAA percent low attenuation area at -950 Hounsfield units, BODE BMI, airflow obstruction, dyspnea and exercise capacity index, FACIT functional assessment of chronic illness therapy fatigue score

subjects. Biomarkers of disease activity are not only needed to accelerate drug development but also to understand the heterogeneity of COPD, which can make it difficult to diagnose and choose the right treatment for each patient. We benchmarked the only FDA approved blood-based biomarker of COPD, plasma fibrinogen, with biomarkers reflecting $\mathrm{BM}$ remodeling by measuring the degradation of COL4A3, by plasma C4Ma3 and TUM. We found that C4Ma3 was highly associated with all-cause mortality and showed to be superior to fibrinogen in predicting mortality since COPD subjects being high in $\mathrm{C} 4 \mathrm{Ma} 3$ had a poorer prognosis than those with low C4Ma3 levels. More interestingly, combining C4Ma3 and fibrinogen was able to improve the prediction of mortality compared to C4Ma3 and fibrinogen alone. In contrast, plasma fibrinogen was related to future self-reported exacerbations whereas C4Ma3 was only related to prior hospitalized exacerbations. The combination of fibrinogen and C4Ma3 was not able to predict future exacerbations either. Furthermore, the data indicated the importance of COL4A3 fragments, since the results for C4Ma3 and TUM were different.

The rate of BM remodeling is proposed to be increased during periods of high disease activity and faster disease progression [19]. Furthermore, it has been shown by Schumann et al. that plasma C4Ma3 levels were increased 

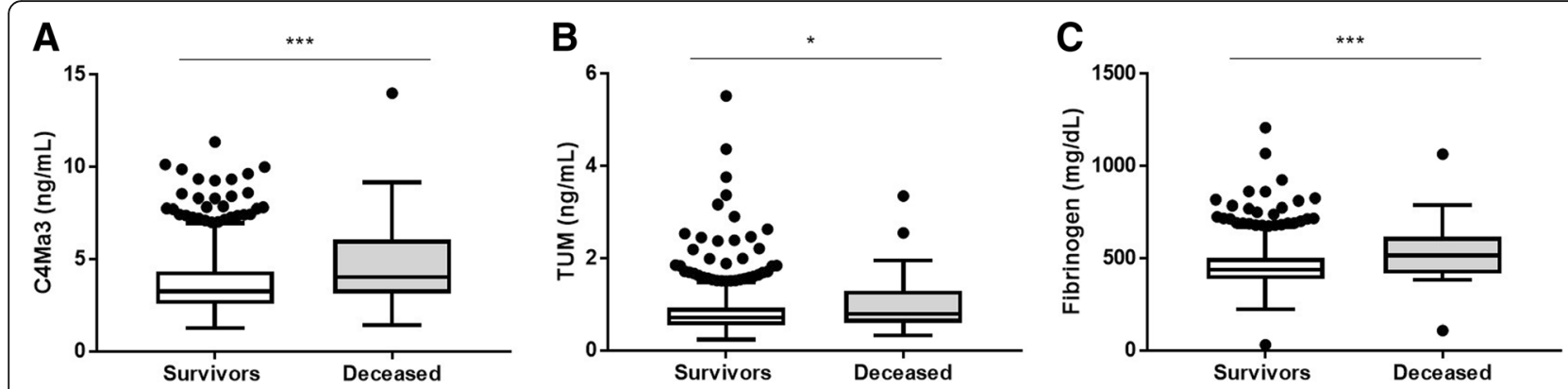

Fig. 3 Plasma C4Ma3 (a), plasma TUM (b) and plasma fibrinogen (c) levels in survivors $(n=954)$ versus deceased $(n=30)$ COPD subjects. Data are shown as Tukey box-and-whiskers plot with a line indicating median, the box indicating the 25th and 75th percentiles (the interquartile range, IQR), and whiskers indicating 1.5 times the IQR. Data points outside this limit are shown by individual points. Asterisks indicate statistical significance: ${ }^{* *} p<0.001$

in COPD subjects from the stable state to the exacerbation and in a hospitalized exacerbation compared to a moderate exacerbation [20]. We showed in this study that plasma C4Ma3 levels were related to previous hospitalized acute exacerbations, which may indicate that plasma C4Ma3 may represent continuous disease activity that results from previous severe events. Plasma fibrinogen was related to hospitalized exacerbations but also self-reported exacerbations, indicating that fibrinogen is more related to exacerbations than C4Ma3. Furthermore, fibrinogen was related to future exacerbations, which was not the case for C4Ma3.

Disease activity is not necessarily correlated with disease severity, patients with severe airflow limitation may not decrease further in $\mathrm{FEV}_{1}$ and thereby not be high in disease activity $[21,22]$. Since $\mathrm{C} 4 \mathrm{Ma} 3$ was proposed as a disease activity marker no correlation was observed with levels of airflow limitation as defined by $\mathrm{FEV}_{1}$.
Fibrinogen showed similar results. Instead, this study showed that plasma $\mathrm{C} 4 \mathrm{Ma} 3$ was an independent predictor of mortality outcome. Using a cut-off level of 5.5 $\mathrm{ng} / \mathrm{mL}$ for C4Ma3, dividing the COPD subjects into high versus low biomarker levels, showed that COPD subjects with high biomarker levels had a poorer prognosis than those with low biomarker levels. Fibrinogen was not able to predict mortality when using the already published cut-off value of $350 \mathrm{mg} / \mathrm{dL}$ in a larger cohort [18] and this may reflect a weaker predictive value.

Fibrinogen is an acute phase soluble plasma glycoprotein, synthesized primarily in the liver and proteolytically cleaved by thrombin into fibrin during blood coagulation, making it a potential biomarker for systemic chronic wound healing [23]. COL4A3 is abundantly expressed in the normal alveolar and glomerular BM, but not in the rest of the body, making C4Ma3 and TUM more specific to the lung. Moreover, COL4A3 has

Table 3 Cox regression in relation to mortality risk

\begin{tabular}{lll}
\hline Parameter & Hazard ratio $(95 \% \mathrm{Cl})$ & $P$ value \\
\hline Age & $1.14(1.0654-1.2201)$ & 0.0001 \\
BMI & $1.01(0.9548-1.0752)$ & 0.6640 \\
BODE & $1.17(0.9828-1.3958)$ & 0.0774 \\
Current smoking & $0.17(0.0564-0.6132)$ & 0.0057 \\
GMWD & $0.99(0.99-1.00)$ & 0.0205 \\
mMRC dyspnea score & $1.55(1.11-2.17)$ & 0.0106 \\
Prior AECOPD & $1.00(0.74-1.37)$ & 0.98 \\
Prior hospitalizations due to AECOPD & $1.52(1.02-2.26)$ & 0.0375 \\
C4Ma3 per SD & $1.65(1.33-2.03)$ & $<0.0001$ \\
C4Ma3 per SD (adjusted for confounders) & $1.57(1.29-1.92)$ & $<0.0001$ \\
TUM per SD & $1.307(1.08-1.58)$ & 0.0064 \\
Fibrinogen per SD & $1.58(1.28-1.94)$ & $<0.0001$ \\
Fibrinogen per SD (adjusted for confounders) & $1.62(1.31-1.99)$ & $<0.0001$ \\
\hline
\end{tabular}

Cox regression in relation to mortality risk. $B M I$ body mass index, $B O D E \mathrm{BMI}$, airflow obstruction, dyspnea and exercise capacity index, $6 M W D$ 6-min walk distance, mMRC modified Medical Research Council score, AECOPD acute exacerbations of chronic pulmonary disease. Confounders included age, current smoking status, $6 \mathrm{MWD}, \mathrm{mMRC}$ score and prior hospitalizations due to AECOPD 

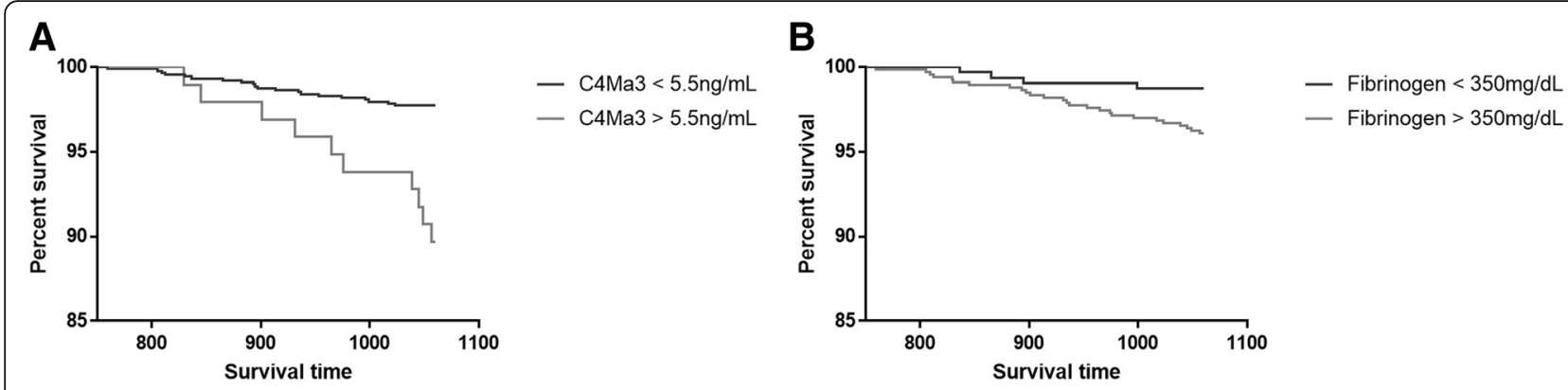

Fig. 4 Kaplan-Meier survival curve analysis using the cut-off $5.5 \mathrm{ng} / \mathrm{mL}$ plasma C4Ma3 (a) and the cut-off of $350 \mathrm{mg} / \mathrm{dL}$ plasma fibrinogen (b)

been shown to inhibit the activation of neutrophils and endothelial cell proliferation, and to induce cell apoptosis by its NC1 domain, tumstatin [24-26]. Tumstatin has been shown to have antiangiogenic effects by blocking the interaction between vascular endothelial growth factor (VEGF) and $\alpha_{\mathrm{V}} \beta_{3}$ integrin leading to apoptosis of proliferating endothelial cells $[27,28]$. This suggests that COL4A3 may be involved in the development of COPD by modulating the inflammatory response or alveolar cell apoptosis [24]. This supports the idea that measuring COL4A3 degradation is interesting for COPD not only by predicting mortality and hospitalized exacerbations but also with a potential role in understanding pathways associated with the pathogenesis of COPD. Notably, the data highlight the importance of knowing the specific fragment that are measured, since the results for $\mathrm{C} 4 \mathrm{Ma} 3$ and TUM were different.

Fibrinogen and the biomarker $\mathrm{C} 4 \mathrm{Ma} 3$ reflect two different aspects of COPD and by combining the two markers COPD patients with chronic wound healing

Table 4 Cox regression using cut-off levels for C4Ma3 and fibrinogen

\begin{tabular}{lcc}
\hline Parameter & Hazard ratio $(95 \% \mathrm{Cl})$ & $P$ value \\
\hline Model 1 (high C4Ma3) & & \\
High C4Ma3 & $5.12(2.28-11.50)$ & 0.0001 \\
Age & $1.11(1.03-1.19)$ & 0.0051 \\
Former smoking & $5.54(1.30-23.60)$ & 0.0206 \\
Model 2 (high Fibrinogen) & & \\
Age & $1.10(1.03-1.19)$ & 0.0079 \\
Former smoking & $4.82(1.12-20.65)$ & 0.0342 \\
mMRC dyspnea score & $1.45(1.03-2.04)$ & 0.0334 \\
Model 3 (high C4Ma3 and high Fibrinogen) & \\
High C4Ma3 & $3.75(1.60-8.80)$ & 0.0024 \\
Age & $1.09(1.02-1.17)$ & 0.0145 \\
Former smoking & $5.03(1.17-21.62)$ & 0.0301
\end{tabular}

Cox proportional hazards regression, enter method. Parameters tested for inclusion in all the models: High biomarker level; Age; smoking status; prior hospitalized acute exacerbations; $m M R C$ modified Medical Research Council dyspnea score and 6MWD 6-min walk distance and remodeling in the basement membrane of the lungs may be identified. We, therefore, speculated that combining the two markers would be a superior prognostic predictor relative to fibrinogen and $\mathrm{C} 4 \mathrm{Ma} 3$ alone. C4Ma3*Fibrinogen improved the prediction of mortality but did not add to the predictive value for self-reported exacerbations.

A key limitation of our study is that data were generated for a subpopulation of the full ECLIPSE study comprising the study participants that progress the least and the most in terms of $\mathrm{FEV}_{1}$ decline over 3 years which may explain the low number of mortality events and reduced statistical power. Despite this, we found significant associations with mortality for C4Ma3 which seems promising and worthy of replication in larger studies. Additionally, baseline samples were not available, shortening the true follow-up time to two years. However, as this was an observational study it should not interfere with the interpretation of the results. Here, we compared a cutoff for C4Ma3 found by ROC curve in the current study with a published cutoff for fibrinogen. To determine if the associations are applicable to the general COPD population and to confirm if C4Ma3 is superior to fibrinogen in predicting mortality, the results should, therefore, be confirmed in an independent cohort.

In conclusion, we were able to compare fibrinogen, C4Ma3 and TUM as biomarkers of disease activity in COPD, alone and in combination. We showed that high levels of plasma C4Ma3 were associated with poorer prognosis and that plasma C4Ma3 levels were related to

Table 5 Cox regression combining C4Ma3 and fibrinogen

\begin{tabular}{lll}
\hline Parameter & Hazard ratio $(95 \% \mathrm{Cl})$ & $P$ value \\
\hline Model 4 (High C4Ma3*Fibrinogen) & & \\
High C4Ma3*Fibrinogen & $5.74(2.65-12.41)$ & $<0.0001$ \\
Age & $1.11(1.03-1.19)$ & 0.0072 \\
Former smoker & $5.37(1.26-22.86)$ & 0.0231
\end{tabular}

Cox proportional hazards regression, enter method. Parameters tested for inclusion in all the models: High biomarker level; Age; smoking status; prior hospitalized acute exacerbations; $m M R C$ modified Medical Research Council score and 6MWD 6-min walk distance 

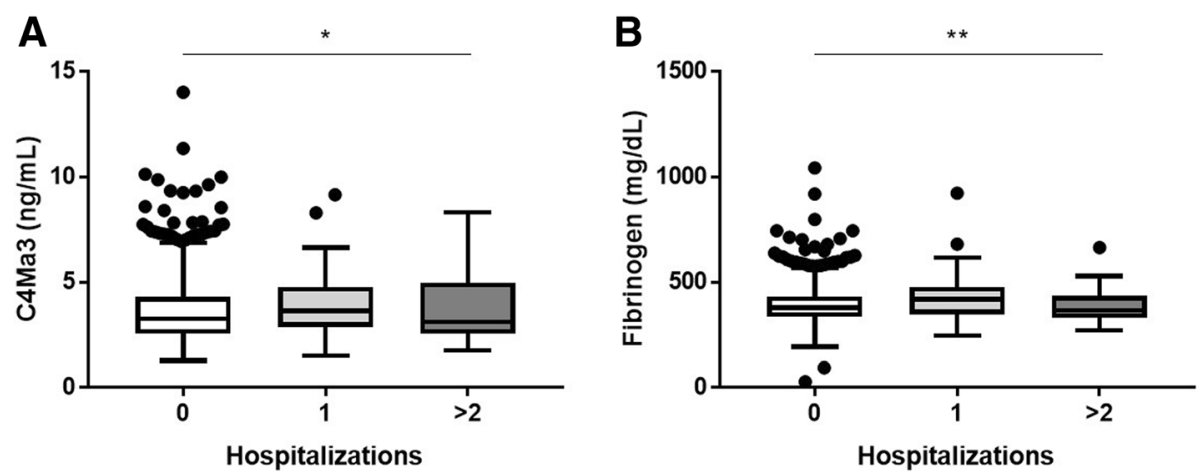

Fig. 5 Plasma C4Ma3 (a) and plasma fibrinogen (b) levels in COPD subjects with no hospitalizations $(n=839)$, one hospitalization $(n=81)$ and more than 2 hospitalizations $(n=28)$ in the year prior to study start. Data are shown as Tukey box-and-whiskers plot with a line indicating median, the box indicating the IQR) and whiskers indicating 1.5 times the IQR. Asterisks indicate statistical significance: ${ }^{*} p<0.05,{ }^{* *} p<0.01$, ${ }^{* * *} p<0.001$ and ${ }^{* * * *} p<0.0001$

hospitalization due to exacerbation prior to study start. Plasma fibrinogen was not related to mortality using the published cut-off value of $350 \mathrm{mg} / \mathrm{dL}$ but showed to be associated with both hospitalized and self-reported exacerbations and future exacerbations. Furthermore, combining C4Ma3 and fibrinogen improved the prediction of mortality, but the combination was not related to future exacerbations. These results indicate that $\mathrm{C} 4 \mathrm{Ma} 3$ may be able to identify COPD subjects with high disease activity and poor prognosis alone or in combination with the current FDA approved biomarker plasma fibrinogen.

\section{Additional file}

Additional file 1: Table S1. Ethics and review boards. (PDF $169 \mathrm{~kb}$ )

\begin{abstract}
Abbreviations
6MWD: 6-min walk distance; AECOPD: Acute exacerbations of chronic pulmonary disease; AUC: Area under the curve; BM: Basement membrane; BMI: Body mass index; BODE: Body-mass-index, airflow obstruction, dyspnea and exercise capacity index; C4Ma3: Biomarker of MMP-mediated degradation of the type IV collagen alpha 3 chain; COL4A3: The alpha 3 chain of type IV collagen; COPD: Chronic obstructive pulmonary disease; ECLIPSE: Evaluation of COPD longitudinally to identify predictive surrogate end-points; ECM: Extracellular matrix; FDA: U.S. Food and Drug Administration; FEV1: Forced expiratory volume in the first second; FVC: Forced vital capacity; GOLD: Global initiative for chronic obstructive lung disease; IPF: Idiopathic pulmonary fibrosis; LLA: Low areas of attenuation below 950 Hounsfield unit; MMP: Matrix metalloproteinases; mMRC: Modified Medical Research Council dyspnea scale; NC1: C-terminal non-collagenous domain of type IV collagen; ROC: Receiver operating curve; SD: Standard deviation; SGRQ: St. George's respiratory questionnaire; TUM: Tumstatin; VEGF: Vascular endothelial growth factor
\end{abstract}

\section{Acknowledgments}

The authors acknowledge all participants, medical, nursing, and technical staff involved in the ECLIPSE study, as well as the technical staff responsible for the biomarker assessments. JV was supported by the NIHR Manchester Biomedical Research Centre.

The Evaluation of COPD Longitudinally to Identify Surrogate Endpoints (ECLIPSE) study, investigators

Ruth Tal-Singer, Bruce E. Miller, Jørgen Vestbo.

\section{Funding}

The ECLIPSE study was sponsored by GlaxoSmithKline (GSK); and this analysis was supported by the Danish Agency for Science, Technology and Innovation; and the Danish Research Foundation. The study sponsor did not place any restrictions about statements made in the final paper.

\section{Availability of data and materials}

The datasets used and/or analyzed during the current study are available from the corresponding author on reasonable request.

\section{Authors' contributions}

Two representatives of GlaxoSmithKline (RT-S, BEM) and one academic (JV), together representing the ECLIPSE study investigators, developed the current study design and concept in collaboration with representatives of Nordic Bioscience (JMBS, SRR, LLA, TMJ, DJL, MAK), they approved the plan for the current analyses, had full access to the data, and were responsible for the decision to publish. All authors read and approved the final manuscript.

Ethics approval and consent to participate

The study was conducted according to the Declaration of Helsinki and Good Clinical Practice guidelines and was approved by relevant ethics and review boards (Additional file 1). Participants provided informed consent.

\section{Consent for publication}

Not applicable.

\section{Competing interests}

JMBS, SRR, TMJ, DJL, and MAK are employees and DJL and MAK are shareholders of Nordic Bioscience. RT-S and BEM are employees and shareholders of GSK. JV has received honoraria for presenting and advising from Astra Zeneca, Boehringer-Ingelheim, Chiesi, GlaxoSmithKline and Novartis, outside the submitted work.

\section{Publisher's Note}

Springer Nature remains neutral with regard to jurisdictional claims in published maps and institutional affiliations.

\section{Author details}

${ }^{1}$ Nordic Bioscience A/S, Herlev, Denmark. ${ }^{2}$ University of Southern Denmark, The Faculty of Health Science, Odense, Denmark. ${ }^{3}$ The Faculty of Health and Medical sciences, University of Copenhagen, Copenhagen, Denmark. ${ }^{4}$ GSK R\&D, Collegeville, PA, USA. ${ }^{5}$ Division of Infection Immunity and Respiratory Medicine, The University of Manchester, Manchester Academic Health Science Centre, and Manchester University NHS Foundation Trust, Manchester, England. 
Received: 14 February 2019 Accepted: 14 March 2019

Published online: 01 April 2019

\section{References}

1. Lozano R, Naghavi M, Foreman K, Lim S, Shibuya K, Aboyans V, Abraham J, Adair T, Aggarwal R, Ahn SY, Alvarado M, Anderson HR, Anderson LM, Andrews KG, Atkinson C, Baddour LM, Barker-Collo S, Bartels DH, Bell ML, Benjamin EJ, Bennett D, Bhalla K, Bikbov B, Bin AA, Birbeck G, Blyth F, Bolliger I, Boufous S, Bucello C, et al. Global and regional mortality from 235 causes of death for 20 age groups in 1990 and 2010: A systematic analysis for the Global Burden of Disease Study 2010. Lancet. 2012;380:2095-128.

2. Miller BE, Tal-Singer R, Rennard SI, Furtwaengler A, Leidy N, Lowings $M$, Martin UJ, Martin TR, Merrill DD, Snyder J, Walsh J, Mannino DM. Plasma fibrinogen qualification as a drug development tool in chronic obstructive pulmonary disease: perspective of the chronic obstructive pulmonary disease biomarker qualification consortium. Am J Respir Crit Care Med. 2016;193:607-13.

3. U.S. Food and Drug Administration. Qualification of biomarker - Plasma fibrinogen in studies examining exacerbations and/or all-cause mortality in patients with chronic obstructive pulmonary disease. 2016. Guidance for Industry. http://www.fda.gov/downloads/Drugs/ GuidanceComplianceRegulatorylnformation/Guidances/UCM453496.pdf.

4. Kalluri R. Basement membranes: structure, assembly and role in tumour angiogenesis. Nat Rev Cancer. 2003;3:422-33.

5. Mariyama M, Leinonen A, Mochizuki T, Tryggvason K, Reeders ST. Complete primary structure of the human alpha 3(IV) collagen chain. Coexpression of the alpha 3(IV) and alpha 4(IV) collagen chains in human tissues. J Biol Chem. 1994;269:23013-7.

6. Khoshnoodi J, Pedchenko V, Hudson BG. Mammalian collagen IV. MicrosC Res Tech. 2008:71:357-70.

7. Hudson BG, Reeders ST, Tryggvason K. Type IV collagen: structure, gene organization, and role in human diseases. Molecular basis of goodpasture and alport syndromes and diffuse leiomyomatosis. J Biol Chem. 1993;268:26033-6.

8. Brown KL, Cummings CF, Vanacore RM, Hudson BG. Building collagen IV smart scaffolds on the outside of cells. Protein Sci. 2017:26:2151-61.

9. Nakano KY, Iyama Kl, Mori T, Yoshioka M, Hiraoka T, Sado Y, Ninomiya Y. Loss of alveolar basement membrane type IV collagen alpha3, alpha4, and alpha5 chains in bronchioloalveolar carcinoma of the lung. J Pathol. 2001;194:420-7.

10. Kalluri R, Shield CF, Todd P, Hudson BG, Neilson EG. Isoform switching of type IV collagen is developmentally arrested in X-linked Aport syndrome leading to increased susceptibility of renal basement membranes to endoproteolysis. J Clin Invest. 1997;99:2470-8.

11. Thannickal VJ, Toews GB, White ES, Lynch JP III, Martinez FJ. Mechanisms of pulmonary fibrosis. Annu Rev Med. 2004;55:395-417.

12. Atkinson JJ, Senior RM. Matrix Metalloproteinase-9 in Lung Remodeling. Am J Respir Cell Mol Biol. 2003;28:12-24.

13. Vestbo J, Anderson W, Coxson HO, Crim C, Dawber F, Edwards L, Hagan G, Knobil K, Lomas DA, MacNe W, Silverman EK, Tal-Singer R. Evaluation of COPD longitudinally to identify predictive surrogate end-points (ECLIPSE). Eur Respir J. 2008:31:869-73.

14. Agusti A, Calverley PMA, Celli B, Coxson HO, Edwards LD, Lomas DA, Macnee W, Miller BE, Rennard S, Silverman EK, Tal-Singer R, Wouters E, Yates JC, Vestbo J. Characterisation of COPD heterogeneity in the ECLIPSE cohort. Respir Res. 2010;11:122

15. Sand JM, Larsen L, Hogaboam C, Martinez F, Han M, Larsen MR, Nawrocki A, Zheng Q, Karsdal MA, Leeming DJ. MMP mediated degradation of type IV collagen alpha 1 and alpha 3 chains reflects basement membrane remodeling in experimental and clinical fibrosis - validation of two novel biomarker assays. PLoS One. 2013;8:1-12.

16. Nielsen SH, Willumsen N, Brix S, Sun S, Manon-Jensen T, Karsdal M, Genovese F. Tumstatin, a Matrikine Derived from Collagen Type IVa3, is Elevated in Serum from Patients with Non-Small Cell Lung Cancer. Transl Oncol. 2018. https://doi.org/10.1016/j.tranon.2018.02.005.

17. Celli BR, Locantore N, Yates J, Tal-Singer R, Miller BE, Bakke P, Calverley P, Coxson H, Crim C, Edwards LD, Lomas DA, Duvoix A, MacNee W, Rennard S, Silverman E, Vestbo J, Wouters E, Agustí A. Inflammatory biomarkers improve clinical prediction of mortality in chronic obstructive pulmonary disease. Am J Respir Crit Care Med. 2012;185:1065-72.
18. Mannino DM, Tal-Singer R, Lomas DA, Vestbo J, Graham Barr R, Tetzlaff K, Lowings M, Rennard SI, Snyder J, Goldman M, Martin UJ, Merrill D, Martin AL, Simeone JC, Fahrbach K, Murphy B, Leidy N, Miller B. Plasma Fibrinogen as a Biomarker for Mortality and Hospitalized Exacerbations in People with COPD. Chronic Obstr Pulm Dis. 2015;2:23-34.

19. Sand JMB, Knox AJ, Lange P, Sun S, Kristensen JH, Leeming DJ, Karsdal MA, Bolton CE, Johnson SR. Accelerated extracellular matrix turnover during exacerbations of COPD. Respir Res. 2015;16:69.

20. Schumann DM, Leeming D, Papakonstantinou E, Blasi F, Kostikas K, Boersma W, Louis R, Milenkovic B, Sand JMB, Wouters EFM, Rohde G, Prat C, Welte T, Tamm M, Karsdal M, Stolz D. Collagen degradation and formation are elevated in exacerbated COPD compared to stable disease. Chest. 2018;154: 798-807.

21. Vestbo J, Rennard S. Chronic Obstructive Pulmonary Disease Biomarker(s) for Disease Activity Needed-Urgently. Am J Respir Crit Care Med. 2010;182:863-64.

22. Bihlet AR, Karsdal MA, Bay-Jensen A-C, Read S, Kristensen JH, Sand JMB, Leeming DJ, Andersen JR, Lange P, Vestbo J. Clinical drug development using dynamic biomarkers to enable personalized health care in COPD. Chest. 2015;148:16-23.

23. Duvoix A, Dickens J, Haq I, Mannino D, Miller B, Tal-Singer R, Lomas DA. Blood fibrinogen as a biomarker of chronic obstructive pulmonary disease. Thorax. 2013. https://doi.org/10.1136/thoraxjnl-2012-201871.

24. Kim KM, Park SH, Kim JS, Lee WK, Cha SI, Kim CH, Kang YM, Jung TH, Kim IS Park JY. Polymorphisms in the type IV collagen alpha3 gene and the risk of COPD. Eur Respir J. 2008:32:35-41.

25. Monboisse JC, Garnotel R, Bellon G, Ohno N, Perreau C, Borel JP, Kefalides NA. The alpha 3 chain of type IV collagen prevents activation of human polymorphonuclear leukocytes. J Biol Chem. 1994;269:25475-82.

26. Maeshima Y, Yerramalla UL, Dhanabal M, Holthaus KA, Barbashov S, Kharbanda S, Reimer C, Manfredi M, Dickerson WM, Kalluri R. Extracellular matrix-derived peptide binds to a $\vee \beta 3$ integrin and inhibits angiogenesis. J Biol Chem. 2001:276:31959-68.

27. Hamano $Y$, Zeisberg M, Sugimoto H, Lively JC, Maeshima Y, Yang C, Hynes RO, Werb Z, Sudhakar A, Kalluri R. Physiological levels of tumstatin, a fragment of collagen IV a3 chain, are generated by MMP-9 proteolysis and suppress angiogenesis via aVB3 integrin. Cancer Cell. 2003;3:589-601.

28. Byzova TV, Rabbani R, D'Souza SE, Plow EF. Role of integrin alpha(v)beta3 in vascular biology. Thromb Haemost. 1998;80:726-34.

\section{Ready to submit your research? Choose BMC and benefit from:}

- fast, convenient online submission

- thorough peer review by experienced researchers in your field

- rapid publication on acceptance

- support for research data, including large and complex data types

- gold Open Access which fosters wider collaboration and increased citations

- maximum visibility for your research: over $100 \mathrm{M}$ website views per year

At BMC, research is always in progress.

Learn more biomedcentral.com/submission 